Journal of Computer Science 4 (4): 298-304, 2008

ISSN 1549-3636

(C) 2008 Science Publications

\title{
Out of Focus Blur Estimation Using Genetic Algorithm
}

\author{
Mohsen Ebrahimi Moghaddam \\ Department of Electrical and Computer Engineering, \\ Shahid Beheshti University, G.C. Tehran, Iran
}

\begin{abstract}
In this study, we have presented a method to estimate out of focus blur that works in noisy images precisely. The presented method has been designed based on two rough estimates of blur function parameters and then the real parameter has been calculated using a genetic algorithm. The structure of out of focus function was analyzed in frequency domain to present a robust method. The method was tested on several degraded images and experimental results were great.
\end{abstract}

Key words: Blur identification, out of focus, genetic algorithm

\section{INTRODUCTION}

Blurring procedure is modeled using following equation in literature ${ }^{[3,13]}$ :

$$
g(x, y)=h(x, y) * f(x, y)+n(x, y)
$$

In Eq. $1 \mathrm{~g}, \mathrm{f}, \mathrm{n}$ and $\mathrm{h}$ are observed image, original image, additive noise (usually Gaussian) and PSF of blurring system, respectively.

The blurring function (h) should be estimated to restore original image by only using the observed image. The procedure to estimate this function is called blur identification $^{[4]}$.

Out of focus blurring is the most famous blurring function that occurs frequently in images. In an imaging system focusing has an important role, because defocusing the lens causes the captured image lost its sharpness and some other features. There are two categorize of image restoration methods, in the first one, restoration method restores original image without exact estimation of blur and noise (these methods usually named Blind Restoration). These methods do not depend on the blur type. The EM (ExpectationMaximization) method belongs to this group of algorithms. Second group uses estimated blur. The restoration process in this group of algorithms is more precise than the first one.

Many researchers have presented methods to estimate Out of focus blur since now. The methods that have been presented since now, do not work precisely in images with high level of noise. E.g the method that was presented in ${ }^{[7]}$, tries to determine the exact location of blurred edges using LSF (Line Spread Function) analysis in spatial domain. It uses the edge information to estimate the out of focus blur parameter. Another method that uses image LSF to estimate out of focus blur is presented $a t^{[16]}$. In this method, the Power Spectrum Equalization (PSE) restoration filter in image form is used to restore original image. This method can be applied only onto small areas of frequency. The method was presented in ${ }^{[14]}$ uses block based edge classification to find amount of out of focus blur. This method works mainly on low and median frequencies, therefore, sharp details are not improved in restoration process.

There are some other methods that work in frequency domain, e.g edge information is employed to find out of focus blur in ${ }^{[11]}$. Extended DCT approach was used to detect edge of Bayer pattern in this method. $\mathrm{In}^{[18]}$, another method is presented to compute image depth. To do this, an out of focus blur is employed and at the first step, its blurring parameter is estimated. The OTF (Optical Transfer Function) is used to estimate blur parameters in this study. Using ML method ${ }^{[20]}$ and wavelet transform $^{[12]}$ are also presented in literature to estimate out of focus blur.

The method presented in $^{[17]}$ is designed to determine, if image is out of focused or not. However, the authors presented a method to estimate motion blur parameters but there is no way to identify out of focus blur. The microscopic images were analyzed to create a focused image in ${ }^{[9]}$. There is no blur identification in this method. However, image fusion is employed, several images using voting manner are combined to create a focused image. The sharpness of image is used as a criterion to find out of focused area in this study. Using Kurtosis as a measurement tool is used in ${ }^{[8]}$ to validate out of focus blur parameter. Each candidate parameter is used to restore image then the Kurtosis value of the restored images is measured. Using this measure the best parameter candidate is selected. 
All of these methods have constraints and limitations.

The most important limitation of them is noise. Also, some of these methods need some extra information or special property about original image to estimate out of focus blur precisely. For example ML method needs priori information about the blur parameter. The methods presented at ${ }^{[12,11]}$ need all objects in the front of a back ground have sharp edges. Existing at least one edge pattern is addressed in ${ }^{[14]}$. Also, there is a limit of out of focus amount in the method presented in ${ }^{[14]}$.

Recently, we have proposed a new method to estimate out of focus blur using a mathematical model of image frequency response ${ }^{[5]}$. This method suffers the additive noise, also its precision decreases when noise increases. Its lowest allowed SNR is about $55 \mathrm{~dB}$. In another work, a noise independent method is proposed by $\mathrm{us}^{[2]}$. However, this method is noise independent, but, it needs some parameters which should be adjusted by user.

In this study, a method is presented to estimate out of focus blur. This method has no constraints and works in each noise level. The proposed method uses the properties of wiener filter restoration and Bessel function to estimate out of focus blur. To identify real parameter, two rough estimates of blur parameter are employed. The blurred image was restored using each rough estimate. Wiener filter is used to restore blurred image. With Regards to the properties of out of focus blur function in frequency domain and structure of wiener filter, a genetic algorithm is proposed to estimate out of focus blur parameter. We have supposed that the noise model is Gaussian with zero mean. Also we presented exhaustive experimental results in statistical form which can be used in method evaluation. The experimental results were satisfactory.

Out of focus blur model: In most cases, the out of focus blur caused by a system with circular aperture can be modeled as follow ${ }^{[1,7,13]}$.

$$
h(x, y)=\left\{\begin{array}{lr}
\frac{1}{\pi R^{2}} \text { if } \sqrt{x^{2}+x^{2} \leq R} \\
0 & \text { otherwise }
\end{array}\right.
$$

In Eq. $2 \mathrm{R}$ is the radius of COC (Circle of Confusion). It has been shown in ${ }^{[19]}$ that an accurate and complex physical model does not result in significantly restoration than this geometric model.

Regarding to Eq. 2 to determine the blurring function it is significant to find $\mathrm{R}$. The frequency response of Eq. 2 which is called OTF (Optical Transfer Function) is defined in Eq. 3 that is based on a Bessel function of the first kind ${ }^{[21]}$ :

$$
H(u, v)=\left[\frac{J_{1}\left(R \sqrt{u^{2}+v^{2}}\right)}{R \sqrt{u^{2}+v^{2}}}\right]
$$

where, $\mathrm{J}$ is the Bessel function of first kind and $\mathrm{R}$ is radius of $\mathrm{COC}$. The Bessel function is defined as:

$$
\mathrm{J}_{\mathrm{a}}=\sum_{\mathrm{m}=0}^{\infty} \frac{(-1)}{\mathrm{m} ! \Upsilon(\mathrm{m}+\mathrm{a}+1)}\left(\frac{\mathrm{x}}{2}\right)^{2 \mathrm{~m}+\mathrm{a}}
$$

The $\Upsilon$ function is defined as follow when $\mathrm{Z}$ is a complex number:

$$
\Upsilon(Z)=\int_{0}^{\infty} t^{(z-1)} e^{-t}
$$

If $\mathrm{Z}$ was a real function definition of $\Upsilon$ is:

$$
\Upsilon(Z)=(Z-1) !
$$

Figure 1 shows the frequency response of Eq. 2 with specified radius and Fig. 2 and 3 show blurred images using out of focus blurring and their frequency response.

\section{Blur estimation method:}

Mathematical foundation: With Regards to Eq. 2 to estimate blurring function, it is enough to estimate the $\mathrm{R}$ parameter that is radius of COC. To do it, some mathematical observations are needed.

Suppose that the radius of COC in Eq. 2 is R. If we consider another blurring function that its parameter is $\mathrm{R}_{1}$, then with regards to Eq. 3 we can conclude that:

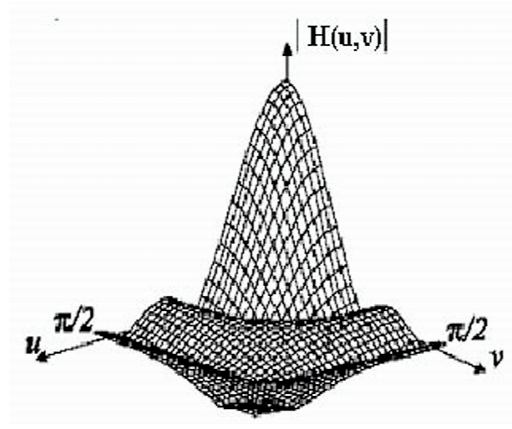

Fig. 1: The frequency response of out focus blur with $\mathrm{R}$ $=2: 5$ pixels 

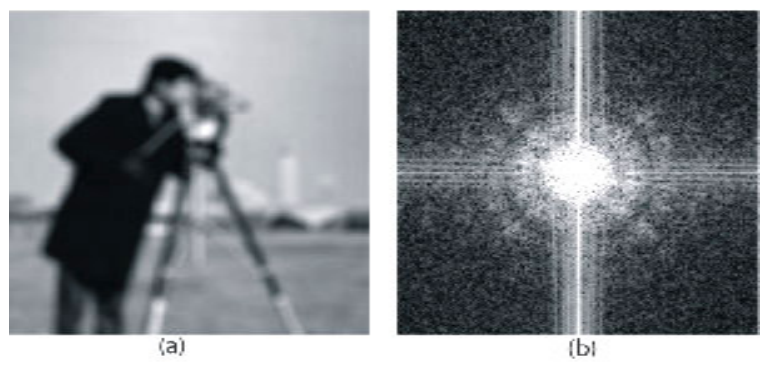

Fig. 2: (a): Camera man image that is degraded using out of focus blur with parameter $\mathrm{R}=10$ pixels with no additive noise (b): Frequency response of (a)

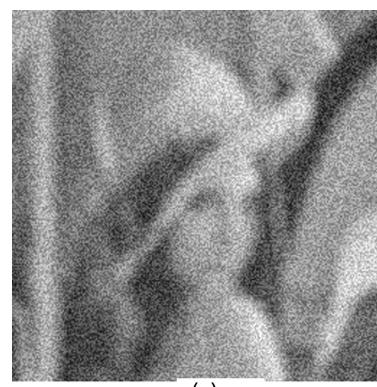

(a)

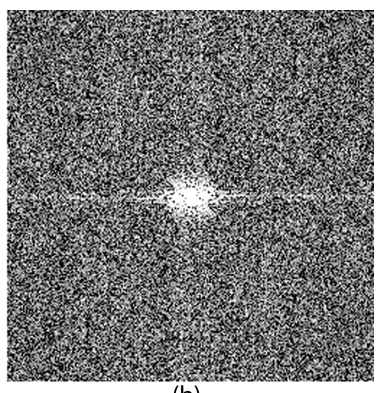

(b)
Fig. 3: (a): Lena image that is degraded using out of focus blur with parameter $\mathrm{R}=12$ pixels and additive noise $(\mathrm{SNR}=40 \mathrm{~dB}),(\mathrm{b})$ : frequency response of (a)

$$
H_{1}(u, v)=\left[\frac{J_{1}\left(R_{1} \sqrt{u^{2}+v^{2}}\right)}{R_{1} \sqrt{u^{2}+v^{2}}}\right]
$$

Using Eq. 3 and 7 we can conclude that:

$$
\frac{H(u, v)}{H_{1}(u, v)}=\left[\frac{J_{1}\left(R_{1} \sqrt{u^{2}+v^{2}}\right)}{R_{1} \sqrt{u^{2}+v^{2}}}\right]
$$

The Eq. 8 concludes the following equation at the frequency center:

$$
\lim _{(u, v) \rightarrow 0} \frac{H(u, v)}{H_{1}(u, v)}=\frac{R}{R_{1}}
$$

If Wiener filter was used to restore degraded image, its equation should be considered.

The Wiener filter is defined by following equation in frequency domain:

$$
\mathrm{W}(\mathrm{u}, \mathrm{v})=\frac{\mathrm{H}^{*}(\mathrm{u}, \mathrm{v})}{\left|\mathrm{H}_{1}(\mathrm{u}, \mathrm{v})\right|^{2}+\mathrm{K}}
$$

Or

$$
\mathrm{W}(\mathrm{u}, \mathrm{v})=\frac{1}{\mathrm{H}(\mathrm{u}, \mathrm{v})} \cdot \frac{\mathrm{H} *(\mathrm{u}, \mathrm{v})}{\left|\mathrm{H}_{1}(\mathrm{u}, \mathrm{v})\right|^{2}+\mathrm{K}}
$$

In these equations, $\mathrm{W}(\mathrm{u}, \mathrm{v})$ shows wiener filter in frequency domain, $\mathrm{H}(\mathrm{u}, \mathrm{v})$ shows the frequency response of degradation function and $\mathrm{K}$ is a constant that models signal to noise ratio ${ }^{[22]}$. As it is shown in Eq. 10 and 11 Wiener filter works in frequency domain, therefore, we should convert equation (1) to frequency domain as follows:

$$
\mathrm{G}(\mathrm{u}, \mathrm{v})=\mathrm{F}(\mathrm{u}, \mathrm{v}) \cdot \mathrm{H}(\mathrm{u}, \mathrm{v})+\mathrm{N}(\mathrm{u}, \mathrm{v})
$$

In Eq. 12, G, H, F and $\mathrm{N}$ show the frequency response of observed image, blurring function, original image and additive noise respectively.

To restore a degraded image it is enough to use the following equation:

$$
\mathrm{F}^{\prime}(\mathrm{u}, \mathrm{v})=\mathrm{G}(\mathrm{u}, \mathrm{v}) \cdot \mathrm{W}(\mathrm{u}, \mathrm{v})
$$

Where, $F^{\prime}, \mathrm{G}$ and $\mathrm{W}$ show restored image frequency response, observed image frequency response and Wiener filter in frequency domain, respectively. Because observed image is already available to restore image we need to estimate $\mathrm{W}(\mathrm{u}, \mathrm{v})$ that is based on $\mathrm{H}(\mathrm{u}$, v) and $\mathrm{K}$. Therefore, it is enough to find frequency response of degradation function $(\mathrm{H}(\mathrm{u}, \mathrm{v}))$ and estimating $\mathrm{K}$ to restore image.

Estimating signal to noise ratio which can be used in Wiener filter to restore original image is addressed in $^{[4]}$. The method presented in ${ }^{[4]}$ employed a genetic algorithm with MSE (Mean Square Error) and IAWE (Image Activity Weighted Error) as its measure to estimate K. However, the presented method $\operatorname{in}^{[4]}$ is suitable for situations that blurring function is identified. Because we are going to estimate the blurring function and signal to noise ration simultaneously, we should employ some other relations. Therefore, if we suppose the corrupted images is degraded by out of focus blur, at first step of blur identification, an arbitrary COC radius $\left(\mathrm{R}_{\mathrm{a}}\right)$ is used to create a degraded function $\left(\mathrm{H}_{\mathrm{a}}\right)$. Then, this degradation function $\left(\mathrm{H}_{\mathrm{a}}\right)$ is used to restore original image, consequently:

$$
F_{a}(u, v)=G(u, v) \frac{\left|H_{a}(u, v)\right|^{2}}{H_{a}(u, v) *\left|H_{a}(u, v)\right|^{2}+K}
$$



that:

With regards to Eq. 14 and 12 we can conclude

$$
\begin{aligned}
\mathrm{F}_{\mathrm{a}}(\mathrm{u}, \mathrm{v})= & (\mathrm{F}(\mathrm{u}, \mathrm{v}) \cdot \mathrm{H}(\mathrm{u}, \mathrm{v})+\mathrm{N}(\mathrm{u}, \mathrm{v})) \\
& \frac{\left|\mathrm{H}_{\mathrm{a}}(\mathrm{u}, \mathrm{v})\right|^{2}}{\mathrm{H}_{\mathrm{a}}(\mathrm{u}, \mathrm{v}) *\left|\mathrm{H}_{\mathrm{a}}(\mathrm{u}, \mathrm{v})\right|^{2}+\mathrm{K}}
\end{aligned}
$$

It is obvious that DC part of degradation function $\left(\mathrm{H}_{\mathrm{a}}(0,0)\right)$ should be 1 , Also, if we assume that additive noise average is zero, by considering the Eq. 9 the following equation can be concluded:

$$
\mathrm{F}_{\mathrm{a}}(0,0)=\frac{\mathrm{R} \cdot \mathrm{F}(0,0)}{(1+\mathrm{K}) \cdot \mathrm{R}_{\mathrm{a}}}
$$

With regards to $\mathrm{H}(0,0)=1$, it is obvious that $\mathrm{F}(0,0)=\mathrm{G}(0,0)$, therefore in the Eq. 16 the $\mathrm{Fa}(0,0), \mathrm{K}$ and $\mathrm{R}$ are unknown parameters, however $\mathrm{Ra}$ and $\mathrm{F}(0,0)$ are known ones. If we solve the Eq. 16 to find $\mathrm{R}$ then:

$$
\mathrm{R}_{\mathrm{a}}^{\prime}=\frac{\mathrm{F}_{\mathrm{a}}(0,0) \cdot \mathrm{R}_{\mathrm{a}} \cdot(1+\mathrm{K})}{\mathrm{G}(0,0)}
$$

Where, $R_{a}^{\prime}$ is the estimated value of $R$ using Ra. If a proper $\mathrm{K}$ can be estimated for wiener filter, $\mathrm{Fa}(0,0)$ can be estimated. Therefore, determining the $\mathrm{R}$ is possible. The equations are presented in this subsection are used to provide blur estimation algorithm in next subsection.

A genetic algorithm to identify blur: At now, a genetic algorithm is proposed, which it can estimate $\mathrm{K}$ and $\mathrm{R}$ simultaneously. To create a proper fitness function, another arbitrary parameter $\mathrm{Rb}$ and its corresponding restored image are needed. With regards to Eq. 16 we can conclude that:

$$
\mathrm{F}_{\mathrm{b}}(0,0)=\frac{\mathrm{R} \cdot \mathrm{F}(0,0)}{(1+\mathrm{K}) \cdot \mathrm{R}_{\mathrm{b}}}
$$

Consequently:

$$
\mathrm{R}_{\mathrm{b}}^{\prime}=\frac{\mathrm{F}_{\mathrm{b}}(0,0) \cdot \mathrm{R}_{\mathrm{b}} \cdot(1+\mathrm{K})}{\mathrm{G}(0,0)}
$$

However, the fitness function is defined as following equation:

$$
\min \text { imize }: \operatorname{Fit}\left(\mathrm{C}_{\mathrm{i}}\right): \operatorname{abs}\left(\mathrm{R}_{\mathrm{b}}^{\prime}-\mathrm{R}_{\mathrm{a}}^{\prime}\right)
$$

Where, $C_{i}$ is a genotype. $R_{b}^{\prime}$ and $R_{a}^{\prime}$ are defined in previous paragraphs. The gentype is defined as a bit string of some zero and one values ${ }^{[15]}$. Here, it is a 16 bit string (2 Byte). The overall structure of proposed genetic algorithm can be introduced as follows:

- Create the initialization population randomly, however, each gentype consists of some random zero or one bits

- Normalize the value of each gentype

- Use the value of each normalized gentype as estimation of $\mathrm{K}$ and apply wiener filter on corrupted image using $\mathrm{Ha}$ and $\mathrm{Hb}$ separately

- With regards to Eq. 17 and 19 , calculate $\mathrm{R}_{\mathrm{b}}^{\prime}$ and $\mathrm{R}_{\mathrm{a}}^{\prime}$

- Select and keep the best $\mathrm{K}$ that minimizes Fitness function

- Cross over and/or mutate the gentype to obtain new generation

- Repeat above steps till finding the best result of $\mathrm{K}$

In this algorithm, the initial population consists of 100 gentype, the scatter crossover with $\mathrm{pc}=0.9$ is used and random mutation with $\mathrm{pm}=0.2$ is employed ${ }^{[10]}$. After running this algorithm, we have found the $\mathrm{K}$ value with regards to optimized $R$. Estimated values of $R_{b}^{\prime}$ and $R_{a}^{\prime}$ shows estimated value of $R$. Therefore, this value can be used as blurring parameter.

\section{RESULTS AND DISCUSSION}

To validate our method: a test bed was created that consists of more than 100 images with different noise variance and degradation function. Standard images like Lena, Baboon, Barbara and etc were used to create the test bed. The images were degraded by out of focus and additive Gaussian noise. The out of focus parameter and noise variances were selected randomly to create each image in the test bed. The interval of random degradation parameter $(\mathrm{R})$ was [2..16] and the random noise variance $\sigma_{\mathrm{n}}^{2}$ was [0.01..0.6]. The noise mean was considered as zero. The resolution of all images in the test bed was $256 \times 256$.

After creating this test bed, our algorithm ran on these images. Table 1 shows some real parameters and their estimated values using our algorithm and Table 2 shows the average error and its standard deviation in our method. The genetic algorithm converged in about 80 epochs in average. Our algorithm was able to estimate degradation parameter in images with high level of noise. Figure 4 and 5 show some blurred image and their corresponding estimated parameter using our method. Figure 6 shows a degraded image and its restored result using wiener filter. The experimental results show our method precision. 
Most of the methods have presented since now, have no exhaustive experimental results in statistical Table 1: Value of some estimated parameter v.s real ones

\begin{tabular}{lll}
\hline Real R & Estimated value & Noise Variance \\
\hline 4 & 4.78 & 0.15 \\
6 & 7.5 & 0.25 \\
8 & 9.7 & 0.32 \\
10 & 10.4 & 0.1 \\
12 & 10.5 & 0.35 \\
14 & 16.3 & 0.4 \\
\hline
\end{tabular}

Table 2: Average and standard deviation of errors in parameter estimation in proposed algorithm

\begin{tabular}{lll}
\hline Error average & Error std & Noise average variance \\
\hline 1.76 & 1.5 & 0.3 \\
\hline
\end{tabular}

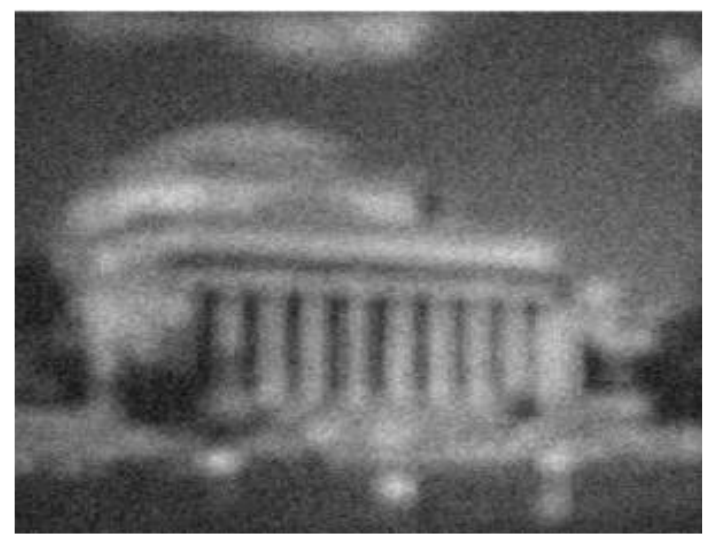

Fig. 4: Columbia picture which was degraded by $\mathrm{R}=11$ pixel and $\mathrm{SNR}=60 \mathrm{~dB}$ pixels. Estimated values for this image using our algorithm were $\mathrm{R}=11.1$ pixels.

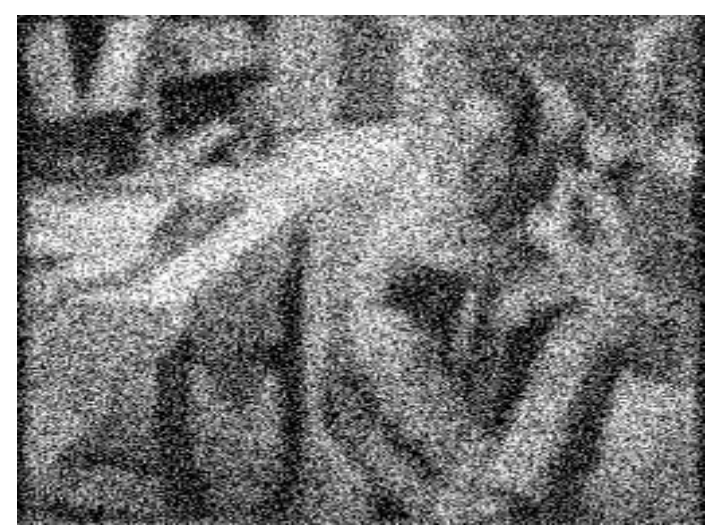

Fig. 5: Barbara picture which was degraded by $R=6$ pixel and $\mathrm{SNR}=35 \mathrm{~dB}$ pixels. Estimated values for this image using our algorithm were $\mathrm{R}=6.04$ pixels form. Most of them were presented the experimental results for one or some images in form of restored image. This makes the comparison between methods

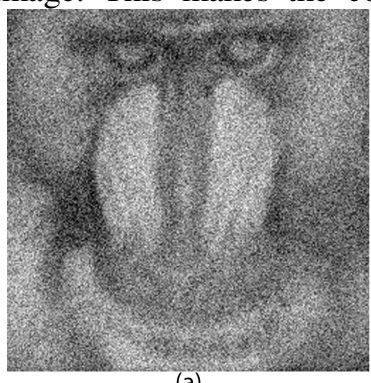

(a)

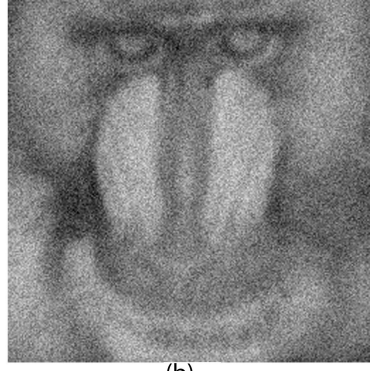

(b)
Fig. 6: (a): Baboon picture which was degraded by $\mathrm{R}=7$ pixel and SNR $=50 \mathrm{~dB}$ pixels. (b): Restored image of (a) using wiener filter and our algorithm

very difficult. However, in this section we have tried to compare our methods with other method in qualitative mode by comparing their constraints and the results presented in corresponding papers.

The method presented in ${ }^{[18]}$ has been tested on both synthetic and real data. RMS of the estimated error in this method was about 0.03 in average. Because this method estimates the out of focus parameter based on image depth, its experimental results showed that monotically increasing the distance of objects in image increases the blur estimation error. There is no such a constraint in our presented method.

$\mathrm{In}^{[20]}$, two images are presented in experimental results and the results of blur estimation for these images are presented. The first image was a blurred sharp text image. It was blurred using $\mathrm{R}=5$ pixels as blurring parameters and SNR $=40 \mathrm{~dB}$ as additive noise parameter. The estimated parameter was very precise in such a way ${ }^{R R}=5$ pixel. But increasing the noise value decreasing the precision of estimation, when the SNR = $20 \mathrm{~dB}$ the estimate parameter were $\mathrm{R}^{\prime}=4.8$ pixel. Our method does not depend on noise theoretically and its precision does not decrease by increasing the noise.

A blur estimation method and a new inverse filter are presented in $^{[11]}$ which its authors did not discuss about noise effects on the precision of their method. It seems the presented method $\mathrm{in}^{[11]}$ works in noise free environments only. $\operatorname{In}^{[14]} 11 \mathrm{~dB}$ improvement in SNR support is reported but there is no numerical result for method precision in its simulation results. Same situations are occurred in $^{[16,17]}$. 
The method presented in ${ }^{[6]}$ needs a rectangular patch with in blurred image and an upper bound of the blur kernel that should be specified by user. These constraints are not available in our method. The lowest allowed SNR in our previous research ${ }^{[5]}$ was about 55 $\mathrm{dB}$, but the presented method has no limitation on the noise level. The method presented in $^{[2]}$ needs user adjustment to find precise answers, however, it uses Besel function attributes. The proposed method needs no adjustment and it can estimate out of focus parameter precisely.

In a overall look, the proposed method outperformed other methods because it has no constraints, its precision is suitable and it supports high level noisy environments.

\section{CONCLUSION}

In this study we have presented a precise and robust method to estimate out of focus blur. In spite of most other presented method since now, our method has no constraints and it can work on all defocused images. This method estimates precisely blur parameters. It uses a genetic algorithm to estimate signal to noise ration and degradation parameters simultaneously. Our method is not dependent to the noise level. To test our method we used some degraded images that additive noise was added to them. The type of image degradation was known and we used our method to estimate its parameter. The experimental results showed our method precision.

In future work we are going to develop a method that can estimate the parameter more precisely using evolutional neural networks.

\section{REFERENCES}

1. Ebrahimi Moghaddam, M., 2008. Out of focus blur estimation using genetic algorithm. In: 15th International Conference on Systems, Signals and Image Processing (IWSSIP), June, pp: 417-420, Bratislava, Slovak.

2. Ebrahimi Moghaddam, M., 2008. A robust noise independent method to estimate out of focus blur. In: 33th IEEE International Conference on Advanced Signal Processing (ICASSP), pp: 12731276, March, LosVegas, USA. DOI: 10.1109/ICASSP. 2008.4517849.

3. Ebrahimi Moghaddam, M. and M. Jamzad, 2007. Motion blur identification in noisy images using mathematical models and statistical measures. Pattern Recog. J., 40: 1946-1957. DOI: 10.1016/j. patcog.2006.11.022.
4. Ebrahimi Moghaddam, M. and M. Jamzad, 2007. Linear motion blur parameter estimation in noisy images using fuzzy sets and power. EURASIP J. Adv. Signal Process., 2007: 8-8. DOI: 10.1155/ 2007/68985,2007.

5. Ebrahimi Moghaddam, M., 2007. A mathematical model to estimate out of focus blur. In: 5th IEEE International Symposium on Image and Signal Processing and Analysis (ISPA), pp: 278-281, September, DOI: 10.1109/ISPA.2007.4383705.

6. Fergus, R. et al., 2006. Removing camera shake from a single photograph. ACM Tans. Graph., 25: 787-794. http://cs.nyu.edu// fergus//papers// deblurfergus.pdf.

7. Wu, S. et al., 2005. An objective out-of-focus blur measurement. In: IEEE 5th International Conference on Information, Communications and Signal Processing ICICS 2005, December 6-9, Bangkok, Thailand, pp: 334-338. http: //ieeexplore.ieee.org/xpl /freeabs all.jsp?arnumber $=1689062$.

8. Li, D. et al., 2005. Blind identification based on kurtosis minimization. In: IEEE International Conference on Image Processing (ICIP), September, volume:1, pp: I-905-908, Italy. DOI: 10.1109/ICIP. 2005.1529898.

9. Shoa, T. et al., 2004. Extracting a focused image from several out of focus micromechanical structure images. In: IEEE International Conference on Acoustics, Speech an Signal Processing (ICASSP), Volume:3, pp: iii-505-508, May, Canada. DOI: 10.1109/ICASSP.2004.1326592.

10. Eiben, A.E. and J.E. Smith, 2003. Introduction to Evolutionary Computing, $2^{\text {nd }}$ edition, ISBN: 9783-540-40184-1, New York, Springer.

11. Vivirito, P. et al., 2002. Restoration of out of focus images based on circle of confusion estimate. In: Proceeding of SPIE 47th Annual Meeting (Applications of Digital Image Processing XXV Conference), pp:408-416, July 7-11, Seattle, WA USA. http:// citeseer.ist.psu.edu/vivirito02restoration.html.

12. Filip Rooms et al., 2002. Estimating image blur in the wavelet domain. In: Proceeding of IEEE international conference on Acoustics, Speech and Signal Processing, Volume:4, pp: IV-41904194.DOI: 10.1109/ICASSP.2002.1004934

13. Lagendijk, R.L. and J. Biemond, 2000. Basic Methods for Image Restoration and Identification. In: Hand Book of Image and Vedio Processing, Chapter B, Academic Press, pp: 125-140. http://citeseer.ist.psu.edu/257185.htm. 
14. Sang Ku Kim et al., 1998. Simultaneous Out-offocus blur estimation and restoration for digital auto focusing system. IEEE Trans. Consum. Elect., 44: 1071-1075. DOI: 10.1109/30.713236.

15. Shan Suthahran et al., 1997. SNR Optimisation using genetic algorithm. In: IEEE International Conference on Image Processing (ICIP), October, USA. DOI: 10.1109/ICIP.1997.647763.

16. Ranjit Bhaskar et al., 1994. An iterative frequencydomain technique to reduce image degradation caused y lens defocus and linear motion blur. In: Geoscience and Remote Sensing Symposium, Surface and Atmospheric Remote Sensing: Technologies, Data Analysis and Interpretation, International, 4: 2522-2524. DOI: 10.1109/IGARSS.1994.399786.

17. Seong-ik Jang et al., 1994. A realtime identification method on motion and out of focus blur for a camera. IEEE Trans. Consum. Elect., 40. Pp:145-153, DOI: 10.1109/30.286409.
18. Mats Gokstop, 1994. Computing depth from out-of focus blur using a local frequency representation. In: Proceeding of IEEE International Conference of Pattern Recognition (ICPR), October, volume:1, pp: 153-158, Israiel. DOI: 10.1109/ICPR.1994.576248.

19. Savakis, E. and H.J. Trussell, 1993. On the accuracy of PSF representation in image restoration. IEEE Trans. Image Process., 2: 252-259. DOI: 10.1109/83.217229.

20. Gardana Pavlovic and A. Murat Tekalp, 1992. Maximum likelihood parametric blur identification based on a continuous spatial domain model. IEEE Trans. Image. Process., pp:496-504. DOI: 10.1109/83.199919.

21. Jan Beimond et al., 1990 Iterative methods for image deblurring. Proc. IEEE, 78: pp:856-883. DOI: $10.1109 / 5.53403$.

22. Gonzalez, R.C. and R.E. Woods, 2002. Digital Image Processing. 2nd Edn. Prentice Hall, USA. 